\title{
Hacktivism and the Male-Only Stereotype
}

Leonie Maria Tanczer

Queen's University Belfast

This is an accepted manuscript version for an article published in the journal New Media \& Society. The final publication is available at SAGE via http://dx.doi.org/10.1177/1461444814567983.

The copyright belongs to the author. 


\begin{abstract}
This research explores hacktivism as a new form of online political activism. It uses qualitative interviews with a gender-equal sample of ten self-defined hacktivists to address issues of gender and the discursive strategies used by males and females to handle the hacktivist community's male-only stereotype. The semi-structured interviews are analysed using Foucauldian discourse analysis (FDA). The analysis indicates that male hacktivists relate to this dominant male-only representation through discursive techniques such as the suppression of gender (Male Oblivious Discourse) or mechanisms of vindication (Male Justification Discourse). Female hacktivists use the accentuation of gender and sexism to counteract male-dominant discourses and establish Female Discourses of Resistance (Emphasis Discourse; Negation Discourse). These gender-related argumentative positions and rhetorical mechanisms demonstrate how the male-only stereotype is created and maintained, and how it affects not only hacktivists' talk and sense-making, but also their identity and the hacktivist actions they perform.
\end{abstract}

Keywords: hacktivism, stereotypes, gender, Foucauldian discourse analysis 


\section{Introduction}

News coverage is currently replete with stories on hacking. However, reports on, for example, the LulzSec member Jeremy Hammond, or the former infrastructure analyst of the National Security Agency Edward Snowden, have contributed to the perception of all hackers being young, white, middle class males. This espouses a biased portrayal and a one-sided account of the community. The present research addresses this male-only stereotype, while focusing exclusively on politically motivated hackers, so-called hacktivists (Jordan, 2002; Taylor, 2005).

Hacktivism is a conflation of hacking and activism (Denning, 2001) and constitutes a form of political activism within a collective action setting (Söderberg, 2013). Hacking relates to computer hacking and comprises activities ranging from gaining unauthorized access to systems or data (Cresswell, 2010), manipulating technology for unorthodox means i.e., (re-)constructing networks (Turkle, 1984), to the production of free software (Coleman, 2013; Kelty, 2008; Postigo, 2012). Activism includes actions taken to promote change in opposition to prevailing powers (Hands, 2011) and is, in the context of hacktivism, closely connected to direct action (Jordan and Taylor, 2004). Thus, hacktivists use the same tools and techniques as hackers, but for political means (Illia, 2003).

The current worldwide attention towards hacktivists, and the latest popularity of the hacktivist collectives Anonymous and LulzSec offer ample opportunity for this 
investigation. Although gender imbalance is also prevalent in the general hacker community (Keller, 1991), the hacktivist scene, due to its social and political motivation, seems appropriate for a gender-centred analysis. Furthermore, the striking underrepresentation of females both in the community and within the existing literature reproduces and enhances gender bias, while it ignores females' contribution to this form of activism.

The present research provides the first discourse analysis on the hacktivist community. It examines the discursive construction of this male-only stereotype through language, and its production and maintenance. Drawing on the public stereotype, I analyse the discourse of politically motivated hackers as part of their own perception and self-categorisation. By adopting a social psychology perspective, I investigate how male and female hacktivists approach this stereotype in their talk and sense-making. In particular, I explore females' self-categorisation within the social and political hacktivist-identity, despite its male-only stereotype.

\section{Theoretical Underpinning and Background}

The research is underpinned by the social identity approach (SIA; Reicher et al., 2010). SIA is subsuming social identity theory (SIT; Tajfel, 1982) and selfcategorisation theory (SCT; Turner et al., 1987). Both are concerned with the identity construction of individuals and groups. Identity comprises the representation of the self in an individual as well as a collective context. Thus, SCT argues for a distinction 
between two types of self-categorisation, meaning personal self (me) versus social/collective self (us) (Ellemers et al., 2002). SIT refers to social identity as a form of collective identity, whereby the abstractness of an individual has moved to a collective level. SIA is crucial for this analysis, as social identity not only plays a role when looking at stereotypes (Haslam et al., 1999), but makes group behaviour such as political action possible (Turner, 1982).

According to Drury (2002), collective action is a site where new discourses, identities, and social relations are constructed. Collective action is defined as any action that is directed towards improving a state of disadvantage of a group as a whole (Wright et al., 1990). It can be performed by collectives and/or individuals (Van Zomeren and Iyer, 2009). When referring to online collective action these activities are conducted through information and communication technologies (Postmes, 2007). ${ }^{1}$ Van Laer (2010) identified that the internet not only facilitates and supports traditional offline activism, but creates new modes of online collective action i.e., hacktivism. Yet, despite the internet's empowering potential, existing inequalities remain (Van Laer, 2007). Although software, such as distributed denial-of-service (DDoS) tools (Sauter, 2013), increase the accessibility of participation in online collective action, the argument is certainly applicable for technically sophisticated forms of hacktivism. Hacktivism can therefore imply elitism in the sense that not everyone has the technical skills to conduct all forms of this activism. Moreover, the argument seems reasonable, considering that 
females express significantly more cautious opinions towards technology than males, and computer anxiety and computer self-efficacy studies reveal a gender gap to the disadvantage of women (Durndell and Haag, 2002; McIlroy et al., 2001; Todman and Day, 2006). These findings highlight the need to investigate the male-only stereotype.

Stereotyping is the categorisation of people into social groups based on common prototypical attributes (Brown, 2010). Differences amongst group members are blurred. Stereotypes are conveyed and reproduced culturally (Hilton and Von Hippel, 1996) and perpetuated by language, leading to their stability and consistency (Wang, 2009). Although stereotypes originate from aspects of social reality (Hoffman and Hurst, 1990), they are not automatically accurate. This is addressed in the present study through evidence of the underrepresentation of women in technology and associated gender stereotypes. The perception of men being responsible for technology is deeply rooted in Western societies, and linked with societies' androcentric, patriarchal culture (Gilman, 1971; Moorman and Johnson, 2003). Technology converges with images of masculinity and power (Faulkner, 2001). Therefore feminist criticism on active-passive duality can be transferred onto it. Women are perceived as being on the receiving, passive end of technology, rather than the creative, active one (Arnold and Faulkner, 1985).

Besides, the male dominance and associated misogyny are part of the existing literature on hacking (Jordan and Taylor, 1998; Nagenborg, 2006; Keller, 1991) and 
other IT-related fields such as gaming (Fox and Tang, 2014; Peck et al., 2011). Turkle (1984) identified an underrepresentation of female hackers, and a male-spirited world which is unfriendly to women. Similarly, Jordan and Taylor (2004: 131) highlight that male and female hackers use technology for different means and explain this by a prevalent 'regressive masculinity' defined by competition, mastery and domination. Due to similarities between the hacker and hacktivist communities, corresponding dynamics are evident in hacktivism (Taylor, 2003; Papadimitriou, 2009). However, an analysis of the effects of the male-only stereotype is, at present, missing. As such, this research transfers the notion of the 'invisible girl gamer' in the gaming community (Bryce and Rutter's, 2002: 244) to examine the invisible female hacktivist to further offer explanations of the emergence and maintenance of the male-only stereotype.

\section{Method}

\section{Participants and Data Collection}

I interviewed a gender-equal, self-selected sample of ten self-identified hacktivists. The sample comprises seven participants from the United States of America, two participants from the United Kingdom, and one participant from Israel. I have neither a background in the hacktivist community nor any prior personal connection to the interviewees. I enlisted participants through recruitment emails sent to IT-related mailing lists, the internet presence of hacktivists and hacktivist collectives, and personal contacts. Additional participants were recruited using snowball sampling. 
The semi-structured interviews were conducted between May and August 2013, either face-to-face or digitally using Voice over Internet Protocol services. I told my participants that the research focussed on stereotypes in general, and I only addressed issues of gender in the last interview questions. This allowed me to assist a narrative production independently of a salient topic, which helped to identify gender-related argumentative positions and rhetorical mechanisms.

\section{Data Analysis}

Based on the epistemology of social constructionism (Burr, 2003), I applied Willig's (2008) version of Foucauldian discourse analysis (FDA). FDA investigates how dominant discourses create and shape subjectivity. It examines points of conflict (Parker, 2013) or counter-discourses (Willig, 2008). Thus, dynamics of resistance within and against hacktivists' dominant male-only discourse are analysed.

The following analysis features extracts from the interviews. Participants are referred to as ' $\mathrm{P}$ ' plus gender ('M' = male, ' $\mathrm{F}$ ' = female), and identifying number (i.e., PM1). My responses are marked as 'I'. The symbol ' $(. .$.$) ' is used to identify negligible$ sections of the interview, '...' signifies pauses, while the symbol '[X]' is used to hide words or phrases which could enable participant identification.

\section{Findings}

Following FDA, the analysis focuses on the ways in which the male-only stereotype is constructed through language. All interviews contained examples of 
gendered verbal behaviour. Moreover, differences in the way male and female hacktivists approached the interviews became apparent. The interview transcripts included both explicit and implicit discourses in the construction of the stereotype and its positions and relations to power. This is particularly striking, as participants were not aware of the focus on gender, and highlights the linguistic creation and maintenance of the male-only stereotype in hacktivism.

I discerned four distinct discourses. They are split into two discourses per gender and heavily affect and interplay with each other. These gendered discourses are (a) the Male Oblivious Discourse, whereby gender was non-existent for men; it leads to Female Discourses of Resistance split into the (b) Emphasis Discourse and (c) Negation Discourse, whereby gender is constantly present; as a result of these, (d) the Male Justification Discourse arose, in which gender was acknowledged, but indirectly classified as subsidiary through the usage of mechanisms of vindication.

\section{Male Oblivious Discourse}

The Male Oblivious Discourse was the most subtle, but most prominent discourse. It intertwines with all following ways of talking about gender. The Male Oblivious Discourse constitutes the male hacktivists' subconscious marginalisation and suppression of female hacktivists. It discursively constructs gender as being a non-issue and therefore non-existent. Males apply this suppression of gender, as they do not take any slightly gender-related aspect into consideration until being specifically asked for it. 
Consequentially, no references to their female colleagues were made, nor did they approach aspects of the male-only stereotype, inequality, or sexism within the community. This became particularly evident in sections of the interview which offered the opportunity to refer to gender or the male-only stereotype, such as when asked to refer to famous real-life or fictional hacktivists. Males only mentioned males, such as 'Jeremy Hammond' (PM5), or organisations, such as 'Wikileaks' (PM1, PM4, PM5), rather than females. Male hacktivists seem therefore oblivious to women due to their position deriving from their own identity as male hacktivists in a male-dominated scene. The Male Oblivious Discourse, furthermore, was represented by a difference in the way men talked. It was distinctive that men referred to 'he' (PM9), 'guys' (PM1) or 'man' (PM5). This aspect shows the assumption that their depicted hacktivist colleagues were also males. Through this mechanism, male hacktivists put themselves in the subject position of being the standard or norm. In accordance with FDA, this allows male hacktivists to act independently from and unaware of gender, giving them a sense of apathy. Their hacktivism is related to masculinity. It is not related to divergent gender identities, such as femininity. This became apparent when one male participant addressed security issues regarding the present research. As exemplified in Extract 1, he tried to introduce me, as a female, to cryptography, and advised me to get help from a male friend. Technical knowledge and being 'a geek' (PM9) is thereby equated with being male. 


\section{Extract 1}

PM9: $\quad$ Find a friend of... who is a geek and ask him ah "How could I do that?". If a geek says "It's easy. Just do this and that. Here's this URL." - Do what he says.

In some cases the Male Oblivious Discourse was accompanied by discourses around power, risk, criminality, and heroisation. However, these discourses were not prominent enough to form separate discourses. Instead they go hand in hand with the ability of oppressing gender and femininity within hacktivism. These discourses build upon discursive constructions, such as hacktivists' 'position to change' (PM6), or their 'power to change' (PM1). Although female hacktivists attempt to convey power in their language, it is less marked. Extract 2 provides a good example of the power available to hacktivists and the relation to a male identity. It resembles a statement used by a hacktivist during a television documentary on Anonymous, in which the collective is likened to being a 'big, strong, buff kid' (Knappenberger, 2012). Anonymous and the hacktivist community are again put on a level with masculinity and power by being 'a small kid who is really muscled' (PM1). 


\section{Extract 2}

PM1: $\quad$ And that's why we are Anonymous - basically we are like ah one ... we are like a small kid who is really muscled and so all full of muscles and he does not even know it yet. It's like he is hitting the wall and is then "wow!" I did that. And that's why I think of Anonymous as one kid, who got all this power but does not even know it yet.

Moreover, this talk around power is evident when hacktivism is compared to 'martial arts' (PM9) and 'Kung-Fu' (PM9). It leads to a heroisation and a quasi-military way of speaking through discursive constructions such as 'we really lost a great man' (PM1), and references to 'our fellows' (PM1).

The Male Oblivious Discourse puts women into the subject position of being an exception. Genders are not represented as equals. Consequentially, women within the hacktivist community act in relation to the male perception of their inconspicuousness. They are unperceived, which gives them, from the male perspective, a sense of helplessness. In contrast to men, women are aware of this suppression mechanism, evident in Extract 3. The participant elaborates on the internal group dynamics as 'men are simply used to play with other men' (PF2), supporting that male hacktivists are 'ignorant' (PF2) and 'oblivious' (PF2) due to being in a 'power group' (PF2). 


\section{Extract 3}

PF2: I actually pay attention to the women around me. (...). I think that people within, people within power-groups often....are completely ignorant of the activities of the people in the nonpower-groups. (...) the men are simply used to play with other men. And all their friends are men. And when they go out and do things - even if there's a women sitting right next to themthey're oblivious, because they only pay attention to the person who is like them. Ahm and it takes a certain sort of...ahm enlightenment to be able to realise, to be part of a power-group and realise that the people outside your power-group exist (...).

Overall, female hacktivists indicate that they, unlike their male hacktivist colleagues, 'actually pay attention to the women' (PF2) and do not suppress gender. Instead, they explicitly refer to the Male Oblivious Discourse, and want to change these power indifferences. This resistance, present in Extract 3, represents the Female Discourses of Resistance, which are going to be outlined hereafter.

\section{Female Discourses of Resistance}

The Female Discourses of Resistance are the outcomes and effects of the male suppression of gender and form a female counter-strategy. They are split into two 
discourses, enabling female hacktivists to deal with the Male Oblivious Discourse: (a) they either self-define as hacktivists, whereby they try to act from within the community against male dominance, or (b) they feel separated from the community and their male colleagues, and therefore struggle with the adoption of the hacktivist term and identity. Both discourses were used interchangeably in the interviews and gender is constantly present. Thus, women emphasise gender to raise awareness of inequalities; they use this accentuation of gender and sexism as discursive resources against the suppression of gender from male hacktivists.

Emphasis Discourse. The Emphasis Discourse is the struggle of females to be seen within the hacktivist community and the public perception. This becomes apparent as female hacktivists approached the issue of gender or women-centric topics such as 'abortion' (PF10) both directly and indirectly in the interviews. Additionally, prior to making gender salient, all women tended to address the male-only stereotype. They recognise that hacktivists are 'usually white male' (PF2) and hacking is generally about 'white male privilege' (PF8). Furthermore, in opposition to males, females named famous real-life or fictional female hacktivists, or they refer to female-related hacktivist incidents such as the 'Steubenville [rape] case' (PF8, PF10). This emphasis of women stands in contrast to the indifference exhibited in the Male Oblivious Discourse.

Further, self-defined female hacktivists speak out against sexism and discrimination. They are actively working internally against these occurrences by being 
‘feminist hacktivists' (PF2, PF8) or doing ‘queer advocacy’ (PF3). Participants highlighted the empowerment they receive through 'acting together, collectively on purpose' (PF8). The collective identity of female hacktivists becomes thereby apparent. One participant indicated particular excitement when reading about other female hacktivists in the news. However, she did not want to 'make too big of a deal of it, because I don't want to make it seem like I am shocked that a girl can do it' (PF10). Extract 4 illustrates these emphasis mechanisms, and the female-centred collective aspect of their hacktivism. Women would work 'with other women' (PF8) and use collaboration for 'anti-rape and anti-harassment activism' (PF8).

\section{Extract 4}

PF8: $\quad$ And from having these kinds of discussions ah with other...women ahm... a lot of us came to the conclusion that... basically what evolved naturally was sharing information and stories about people in our field, about people in hackerspaces and hacker culture and basically open-tech and open-culture ahm and finding out who the creeps were, finding out the open secrets ahm...who is another activist who who ahm...is a rapist or is ahm who - you know - what guys in positions of power are doing stuff that some people know about, but nobody is talking 
about - it's not open. So we did a lot of, so we're doing a lot of anti-rape and anti-harassment activism.

Additionally, similar to Extract 3, Extract 4 refers to the power position of males and highlights the Male Oblivious Discourse, when saying that 'nobody is talking about' (PF8) sexism. Within the Emphasis Discourse, female hacktivists perceive the community as modifiable. Gender is actively brought to attention to counteract the female suppression by males and the public. Women take up the subject position of being whistleblowers and opponents of this inequality. In comparison, male hacktivists are perceived as protagonists, causing suppression and discrimination.

Through their collective activism as women for women, female hacktivists achieve a sense of empowerment and agency. In contrast, they also assign a sense of responsibility to males. It becomes obvious that the male-only stereotype affects females' talk and sense-making. This has an effect on what females are doing in terms of their hacktivism.

According to references from female hacktivists, males act more 'destructive[ly]' (PF2) or 'defensive[ly]' (PF7) in their hacktivism. This was also acknowledged by one male hacktivist saying that males are 'more likely to a negative behaviour' (PM9). Women predominantly opposed destructive and illegal hacktivism such as DDoS attacks and see it as a 'really unhelpful act' (PF2). According to female 
participants women tend to use hacktivism more constructively as they are 'iffy' (PF10) when it comes to crossing legal borders. Thus, female hacktivists perceive hacktivism as 'support' (PF2, PF3, PF8, PF10) for others and especially for other women.

The Emphasis Discourse enables females to still associate strongly with their male colleagues. This gives them the feeling of being part of the hacktivist community as they 'have to push less to be listened to' (PF3). Some female hacktivists therefore agree that sexism is far better handled in the hacktivist rather than the hacker community (PF3, PF10).

\section{Extract 5}

PF3: $\quad$ Ahm I think that people are more socially aware of gender roles though. And also more comfortable in calling them out. Ahm... which is exciting. I feel more...I feel more listened to and I have to push less to be listened to when in hacktivist settings than in hacker settings.

However, though hacktivists perceive that there is a 'higher ratio of women hackers being hacktivists then male hackers being hacktivists' (PF2), this has not led to a fair and equal sphere within the community, which will be discussed in the Negation Discourse. 
Negation Discourse. The Negation Discourse originates from the struggle of female participants to be acknowledged as hacktivists. It leaves women in an outsider position. Despite the fact that the interviewed participants were self-defined hacktivists, there were sections in the interviews which indicated that (a) female hacktivists have to justify their identity so as not to be negated by their male colleagues, and (b) some females simply reject the term and identity due to the trouble of being recognised as a hacktivist. The latter became evident in an email I received (Extract 6). The female hacker and activist argued that she does not identify with the term and identity hacktivist, as males are in power to classify who is or is not a real hacktivist and because the activism 'actually needed, isn't recongized as "real hacking” in hacker culture' (Email).

\section{Extract 6}

Email: $\quad[\mathrm{X}]$ forwarded your email to a list i $[$ sic $]$ am on. i identify as an activist, and i identify as a hacker, but i don't identify as a hacktivist - because the activism i have found is actually needed, isn't recognized as "real hacking" in hacker culture - eg, [sic] teaching everyone at occupy how to use wordpress, or teaching sewing classes at a makerspace in a poor neighbourhood... Skills that are categorized as "feminine" are not thought of as real work 
in our society, so because of systemic social prejudice both within the hacker culture and society at large, by definition women are often not hacktivists.

Extract 6 poses issues around skills, femininity versus masculinity and power. Thus, this Negation Discourse is about a fight for control within the hacktivist space and male hacktivists' prerogative of interpretation of who and what is real. The majority of female hacktivists referred to the Negation Discourse with strong and figurative words such as 'battle' (PF2, PF3) or 'shouting and harrowing' (PF2). Within this discourse female hacktivists 'fight on two fronts' (PF8), meaning both 'for their activism and against the discrimination from male hacktivists' (PF8).

The hacktivist community is thereby perceived as being about 'elites' (PF3), and closed to women through a form of 'gate-keeping' (PF8). Women have to 'prove themselves' (PF10) against male standards. Thus, they are not real hacktivists 'unless they have a particular technological skill set' (PF8). Therefore a male 'policing of... of who gets to be a hacker' (PF8) is in place. This leads to a power inequality between genders.

Contrary to the previous Emphasis Discourse shown in Extract 5, whereby women tend to feel more listened to in the hacktivist setting, they speak in the Negation Discourse about the hacktivist community as a 'hostile environment' (PF8). Dynamics 
of exclusion are equated and compared with the hacker scene and show how participants draw on the interplay of both fields. The female perception is that their male colleagues conceive the hacktivist area as a male-only space 'where they can be themselves' (PF10). Hence, female participants argue that 'there is a lot of misogyny' (PF10). The Negation Discourse reflects a struggle of women to (a) enter (Extract 6), and (b) remain within the community (Extract 7). The following Extract 7 demonstrates the problems females face when being part of the hacktivist scene and receiving fame. They are degraded, receive 'personal commentary' (PF8), and are evaluated solely on the basis of their gender.

\section{Extract 7}

PF8: $\quad$ Yeah. I think the result of...ahm...getting that attention is usually pretty negative for women. You get more...ahm...personal commentary, scrutiny about the way you look or your sex life ahm...than you would if you were a man. So, it's not something that is rewarding.

Moreover, females addressed the difficulty of gaining reputation. This becomes particularly evident when they have to work with males both in their hacktivism and in the IT business in general. One participant was asked by an employer to 'pretend that 
you are the secretary' (PF2), despite the fact she was in charge of a project. Another woman feels that if she works with males other people do not see her 'contributions as legitimate' (PF7), because 'then really he is the real one, and I am just helping' (PF7). Their gender is used to exclude them from gaining esteem. The Negation Discourse of females was also complementary with the males' behaviour 'of showing off' (PF7) and 'being macho' (PF8). According to one female participant, especially new male community members use sexism as 'they're trying to prove themselves' and want to present themselves as 'tough and really funny' (PF10).

Besides the struggle to enter and act within the hacktivist community, female hacktivists also tend to feel the need to justify or question their hacktivist identity (Extract 8). For example, one participant revealed that she had not self-defined as a hacker until a male colleague actually 'called [her] a hacker' (PF7). This further adds to the male ability to grant permission to be included into the community. On the basis of this, women vindicated their hacktivism far more than males.

\section{Extract 8}

PF3: $\quad$ Ah, so I don't know that I am - honestly. Ahm I spend a lot of time with people that are. I don't code. I am not a hacker in the traditional sense. (...). And of course you can twist that fuzzy line as much as you want but ahm... because most of what I do 
is... augment the abilities of people who are hacktivists, so I suppose I am also one.

Interesting is a reference made by a male hacktivist who justified why he mentioned a particular woman, when asked specifically to name female hacktivists (Extract 9). He thereby picks up on the male prerogative of interpretation and outlines a hierarchy based on hacktivist skills and methods when talking about 'hardcore hackers' (PM9). This corresponds to Extract 6 where females felt excluded because they would not be considered as real hackers.

\section{Extract 9}

PM9: $\quad$ Now, some people like the hackers who call themselves "hardcore hackers" would go "Ah, she's no hacker! She's just using technology in a nice way!” - ahm...I count her as a hacktivist.

Hence, within the Negation Discourse, women experience their male hacktivist colleagues as the authority, while they feel excluded. This subject position within this discourse permits males a sense of power, giving them a position to define and categorise who is or is not part of the hacktivist community. In comparison, women 
have a sense of unacceptance in their own subject position. They are easily neglected as hacktivists from a male point of view, which can result in women refusing the identity and the term hacktivist (Extract 6). This mechanism adds to the perceived male-only characteristic of the scene. It is therefore important to address the last evolving discourse, which builds upon all of the previously discussed discourses, and enables male hacktivists to deal with the male-only stereotype of their community.

\section{Male Justification Discourse}

The Justification Discourse is the male counter-mechanism to cope with the Female Discourses of Resistance and is a reaction to their own Male Oblivious Discourse. As a result, the Male Justification Discourse can be perceived as a Discourse of Resistance of males. Thus, when I explicitly asked male participants to elaborate on issues of gender and sexism, the troubling nature of the male-only stereotype for the male part of the community became more evident.

Talking about the males' own previously used Male Oblivious Discourse was frequently accompanied by justifications, explanations, stuttering or an apology. The Male Justification Discourse builds upon discursive constructions such as 'but' (PM4, PM9), 'unfortunately' (PM5, PM6), 'don't get me wrong' (PM5), 'we not' (PM1), 'hopefully' (PM6), and 'I think' (PM5, PM6). This has the effect that gender, the maleonly stereotype, and sexism within the community are acknowledged by male hacktivists, but are indirectly classified as subsidiary as males use discursive techniques 
to vindicate themselves (mechanisms of vindication), exemplified in Extract 10. Pauses and 'ahm' (PM4) are indication for the difficulty the participant faces when responding.

\section{Extract 10}

PM4: There's a gender bias but also...there are several levels here. There's also the fictional portrayal ahm and then there's - like the hacktivists - most of the people I work for in $[\mathrm{X}]$ and when I was at $[\mathrm{X}]$ were women. (...).

I: $\quad$ But why do you think did it happen that you just raised males?

PM4: $\quad$ In terms of? - Well, that was, that was in particular you were...ahm...you were ahm - Well, first you were talking about fictional examples, and like I said now - fictional examples I'd be hard-pressed so I can do that once I think about it. Yeah, it's hard - you hard-pressed defined fictional examples of ahm...of ahm...hacktivists. But ahm...

I: I don't wanna put you on the spot. It's like...

PM4: But then...in terms of... - yeah I think in terms of ahm popular conception of...in that - there's definitely a huge gender bias. But I think in like the people actually doing stuff - more than just Anonymous - people like actually doing stuff - there's a lot 
more diversity than... than there's popular awareness about.

Despite the fact that all male hacktivists acknowledge the male-centred nature of hacktivism when specifically asked about it, they feel the need to highlight aspects of defence and self-protection (Extract 10). Other mechanisms of vindication are argumentations around the problem of identification. Female hacktivists would not officially define themselves as hacktivists or 'wave any flags of activism' (PM9). This ties on the previous Negation Discourse of females. The male participants use this to accentuate that this 'makes us...less notice women' (PM9), putting themselves in a less blameable state. Moreover, male participants emphasised that they try to reflect their own 'privileged position' (PM5), or that other 'efforts have gone into changing' inequalities (PM6).

Due to these mechanisms of vindication, which should depict reflectiveness, men refer to the fact that hacktivism constitutes a more women-friendly environment. This was already acknowledged by females in the Emphasis Discourse (Extract 5). Male participants perceive the community as 'self-correcting' (PM4). Nonetheless, mechanisms of vindication can stretch as far as some male participants would argue that gender 'doesn't matter' (PM9) in the hacktivist scene, because 'everybody is equal' (PM9), or that males perceive the problem of sexism as 'exaggerated' (PM9) by some 
'fanatic feminists' (PM9). These are techniques to supersede the prior Male Oblivious Discourse and the male dominance of the community.

Regardless, the Male Oblivious and Justification Discourses should not be misunderstood as discourses of active sexism. Instead, the Male Justification Discourse provides evidence of the subconscious discursive strategies applied to deal with the males' position within the hacktivist community. Here, gender is used to demonstrate concern. The construction of gender as subsidiary requires males to behave as responsible actors and consider the consequences of their actions. Male hacktivists put themselves into the subject position of being defenders. They justify and take responsibility for the inequality and women's wellbeing in the community. It gives them a subjective experience of a sense of guilt.

Contrary to this, the Male Justification Discourse locates women in the subject position of a prosecutor, particularly evident in Extract 11, when saying 'that even men who think of themselves as being pro-women will often do exactly the behaviours that keep women out of their groups' (PF2). Females impeach males' perception of gender inequality and demand recognition. This was already evident in the Female Discourses of Resistance. However, within the Justification Discourse the men finally recognise this claim of women, as they implement, for instance 'specific anti-harassment policies' (PM5) at hacker and hacktivist conferences. This discourse puts women into the subjectivity of having a sense of urgency to act. Women demand recognition from their 
male colleagues and to stop 'misogynist insults' (PF8, PF10). Female hacktivists criticise some forms of mechanisms of vindication used in the Male Justification Discourse when saying that they receive 'tons of excuses' (PF2). They further emphasise that male hacktivists are often 'not aware' (PF2) of their behaviour (Extract $11)$.

\section{Extract 11}

PF2: $\quad$ Ahm the fact that the ahm...the fact that even men who think of themselves as being pro-women will often do exactly the behaviours that keep women out of their groups. And when you confront them with it they will have tons of excuses why, reasons why, ahm or flat out denying. Like: "No, I didn't do that". "You so did that!" - "No, no, no. I would never do" - "You did that. Like all the women in the room saw you do that. You are not aware of your own behaviour".

Yet, both female and male hacktivists agree in the interviews that sexism and techniques such as Male Justification Discourse develop from problems of society at large, due to socialisation and education. They acknowledge that issues such as sexism 
and the male-only stereotype are slowly changing, especially as female hacktivists perceive themselves as people 'who break those stereotypes' (PF10).

\section{Discussion}

This research sought to explore how the male-only stereotype affects the selfperception and discourse of politically motivated hackers. The four discourses arising from the discursive object gender allowed for the examination of how hacktivists' gender and thereby particular positions within the hacktivist community construct gender-related argumentative positions and rhetorical mechanisms. The present analysis indicates how the male-only stereotype (a) is created and maintained i.e., through the suppression of gender and mechanisms of vindication from male hacktivists; (b) affects hacktivists' talk and sense-making i.e., there are gendered discursive resources and techniques such as dynamics of resistance within the talk of female hacktivists; (c) affects males' and females' own social identity and the hacktivist actions they perform i.e., females feel the need to accentuate their gender and the prevalent sexism, leading to different reasons for male and females hacktivist involvement. The research therefore outlines mechanisms which create and sustain the male-only stereotype in hacktivism.

The Male Oblivious Discourse contributes to research of a common Western dynamic, which is the suppression of gender and women both in our language (Holmes and Meyerhoff, 2008) as well as in current societal structures (Coffee, 2013). The usage of 'men' and 'guys' to describe collectives, or the frequent use of 'he' in everyday 
speech highlights this. In particular, feminist linguists criticise the male-centred nature of language, as it influences societal consciousness, affects the way society perceives its environment, and shapes societal ideas about men and women (Pusch, 1990). Parker (1992) argues that discourses facilitate and limit what can be said by whom, where and when. Thus, the fact that males do not speak about gender and women, implies that males do not consider females in their own perception and identity of hacktivists.

However, language not only has the power to affect public awareness, but also represents power structures. The analysis exemplifies ways of defending the societal status quo. This became particularly evident in the two male discourses identified here, as men indirectly put themselves in the position of being the norm. Research conducted by the University of Nevada identified in an analysis of 352 front-page stories from The New York Times that reporters quoted 3.4 times as many male as female sources (Layton and Shepard, 2013). This demonstrates how the public appearance is receptive to males, while females are excluded from this public sphere.

To thwart this process, females follow Foucault's (1977) argument that power and powerful discourses are always intertwined with resistance. The exposure of these Discourses of Resistance is essential to challenge dominance (Eamonn, 2004). Female Discourses of Resistance are the outcome of the male suppression of gender and the prevalence of masculinity in hacktivists' perception and talk. Females counteract this by making their gender discursively present. Through these mechanisms, women achieve 
agency and empowerment. Females either use the Emphasis Discourse to act in a state of acceptance against this male-only stereotype from within the community, or they put themselves into an outsider position when using the Negation Discourse.

Despite the fact that in the Male Justification Discourse gender is used by males to demonstrate concern, they also apply mechanisms of vindication. These findings are similar to a conversation analysis of men's denials of violence towards women. Stokoe (2010: 59) identified that suspects' denials are based on the argumentation 'that they are not the kind of men' who would use violence. Likewise, male hacktivists discursively refer to their own identity as self-reflective men. Thus, they implement anti-harassment policies at hacker conferences, or they make claims about their character, disposition, and identity to justify their position within the discourse.

Building on the existing literature of SIA (Reicher et al., 2010), the gained insights demonstrate the importance of identity. Within both male and female discourses the hacktivist identity is central for their argumentation. However, the women's emphasis on gender indicates that their social identity as hacktivists is linked with their social identity as females. Depending on the social identity which is made salient, they either identify as women who are hacktivists i.e., when gender is salient or conversely hacktivists who are women i.e., when hacktivism is salient.

Moreover, it is interesting to note that women were inclined to refer more to their female hacktivist colleagues then men. Research beyond hacktivism demonstrates 
that gender is more salient for children when having a family background with more members of the opposite sex. These children tend to mention their gender more often when describing themselves (McGuire and Padawer-Singer, 1976). Similarly, gender attitude Implicit Association Tests' show that women's ingroup bias is stronger than men's (Rudman and Goodwin, 2004). This research provides interesting thoughts for the present study, highlighting that the construction of gender-related discourses and identities may extend beyond the hacktivist community.

The importance of gender, further, provides the basis for females' hacktivist actions, exemplifying the discursive connection of social identity with political identity (Huddy, 2001) and adding to collective action research. The findings show that although hacktivists in general are motivated to work against existing inequalities, women within the community are themselves facing power struggles and are lacking acknowledgement. This demonstrates that politically motivated movements who act against power dynamics are themselves not immune to forms of discrimination. Similar mechanisms were found in research on the suppression of bisexuals within the LGBT community (Ault, 1996; Rust, 1993) or erased women of colour within the feminist movement (Crenshaw, 1989).

In closing, research such as the present study offers opportunities for broader societal insights. In understanding how stereotypes discursively manifest and affect people's talk, science and society can attenuate stereotypic thinking. To ensure women a 
place within male-centred areas, it is crucial that both the way society talks about gender, as well as the factual gender imbalance, change. Mechanisms or publications highlighting females, such as Gürer's (2002) review on influential female computer scientists, can accentuate their contributions in a broader public discourse and could counteract the suppression by challenging the 'unicorn problem' of women (Kühn, 2012: 7).

Hacktivism can thereby play an important role in opening up the whole spectrum of IT (Taylor, 2003). As hacktivism broadens the pure technical focus of hacking to hacking for social concerns, it can allow more women to enter technological fields. Therefore, the male's commitment to allow women space in the community and to provide an open environment is important. Hence, whilst further research is clearly required to explore this new form of political activism, the present analysis demonstrates how the male-only stereotype is created and maintained. Furthermore, it indicates how the male-only stereotype not only affects hacktivists' talk and sensemaking, but also how it influences males' and females' own identity and the hacktivist actions they perform. 


\section{Endnotes}

${ }^{1}$ Although this study remains within the framework of collective action research, it acknowledges the theoretical contributions of social movement literature. 


\section{References}

Arnold E and Faulkner W (1985) Smothered by Invention: Technology in Women's Lives. London: Pluto Press.

Ault A (1996) The dilemma of identity: Bi women's negotiations. In: Seidman S (ed) Queer Theory/ Sociology. Oxford: Blackwell, 311-330.

Brown R (2010) Prejudice: Its Social Psychology. Oxford: Blackwell.

Bryce J and Rutter J (2002) Killing Like a Girl: Gendered Gaming and Girl Gamers' Visibility. Tampere: Tampere University Press.

Burr V (2003) Social Constructionism. London: Routledge.

Coffee AMSJ (2013) Mary Wollstonecraft, freedom and the enduring power of social domination. European Journal of Political Theory 12(2): 116-135.

Coleman EG (2013) Coding Freedom: The Ethics and Aesthetics of Hacking. Princeton: Princeton University Press.

Crenshaw K (1989) Demarginalizing the intersection of race and sex: A Black feminist critique of antidiscrimination doctrine, feminist theory and antiracist politics. The University of Chicago Legal Forum: 139-168.

Cresswell J (2010) Oxford Dictionary of Word Origins. Oxford: Oxford University Press.

Denning DE (2001) Activism, Hacktivism, and Cyberterrorism: The internet as a tool for influencing foreign policy. In: Arquilla J and Ronfeldt D (eds) Networks and Netwars: The Future of Terror, Crime, and Militancy. Santa Monica: Rand, 239-288.

Drury J (2002) When the mobs are looking for witches to burn, nobody's safe': Talking about the reactionary crowd. Discourse \& Society 13(1): 41-73.

Durndell A and Haag Z (2002) Computer self efficacy, computer anxiety, attitudes towards the Internet and reported experience with the Internet, by gender, in an East European sample. Computers in Human Behavior 18(5): 521-535. 
Eamonn C (2004) Power, Discourse, and Resistance: A Genealogy of the Strangeways Prison Riot. Aldershot: Ashgate Publishing.

Ellemers N, Spears R and Doosje B (2002) Self and Social Identity. Annual Review of Psychology 53(1): 161-186.

Faulkner W (2001) The technology question in feminism: A view from feminist technology studies. Women's Studies International Forum 24(1): 79-95.Foucault M (1977) Language, Counter-Memory, Practice: Selected Essays and Interviews. Oxford: Blackwell.

Fox J and Tang WY (2014) Sexism in online video games: The role of conformity to masculine norms and social dominance orientation. Computers in Human Behavior 33(1): 314-320.

Gilman CP (1971) Our Androcentric Culture: Or the Man-made World. New York: Johnson Reprint.

Graham LJ (2011) The Product of Text and 'Other' Statements: Discourse analysis and the critical use of Foucault. Educational Philosophy and Theory 43(6): 663-674.

Gürer D (2002) Pioneering women in computer science. ACM SIGCSE Bulletin 34(2): 175-180.

Hands J (2011) @ Is for Activism: Dissent, Resistance and Rebellion in a Digital Culture. London: Pluto.

Haslam SA, Oakes PJ, Reynolds KJ and Turner JC (1999) Social identity salience and the emergence of stereotype consensus. Personality and Social Psychology Bulletin 25(7): 809-818.

Hilton JL and Von Hippel W (1996) Stereotypes. Annual Review of Psychology 47(1): 237-271.

Hoffman C and Hurst N (1990) Gender stereotypes: Perception or rationalization? Journal of Personality and Social Psychology 58(2): 197-208.

Holmes J and Meyerhoff M (2008) The Handbook of Language and Gender. Oxford: Blackwell. 
Huddy L (2001) From social to political identity: A critical examination of social identity theory. Political Psychology 22(1): 127-156.

Illia L (2003) Passage to cyberactivism: How dynamics of activism change. Journal of Public Affairs 3(4): 326-337.

Jordan T (2002) Activism!: Direct Action, Hactivism and the Future of Society. London: Reaktion Books.

Jordan T and Taylor P (1998) A sociology of hackers. The Sociological Review 46(4): 757-780.

Jordan T and Taylor PA (2004) Hacktivism and Cyberwars: Rebels with a Cause? New York: Routledge.

Keller L (1991) Machismo and the Hacker Mentality: some personal observations and speculations. In: Lovegrove G and Segal B (eds) Women into Computing: Selected Papers 1988-1990. New York: Springer, 66-71.

Kelty CM (2008) Two Bits: The Cultural Significance of Free Software. Durham: Duke University Press.

Knappenberger B (2012) We are Legion: The Story of the Hacktivists. USA: Luminant Media.

Kühn LM (2012) Heterosexismus Hacken. Spannungsfelder und Potenziale zum Aufbruch von Geschlechterverhältnissen in der Hacker*szene. Seminar Paper, PhilippsUniversität Marburg, Marburg.

Layton A and Shepard A (2013) Lack of female sources in NY Times front-page stories highlights need for change. Poynter, 16 July 2013.

McGuire WJ and Padawer-Singer A (1976) Trait salience in the spontaneous selfconcept. Journal of Personality and Social Psychology 33(6): 743-754.

McIlroy D, Bunting B, Tierney K and Gordon M (2001) The relation of gender and background experience to self-reported computing anxieties and cognitions. Computers in Human Behavior 17(1): 21-33.Moorman P and Johnson E (2003) Still a Stranger here: Attitudes among Secondary School Students Towards Computer Science. ACM SIGCSE Bulletin 35(5):193-197. 
Nagenborg M (2006) Hacker - Der Computer als Werkzeug und Symbol der Macht. In: Treibel A, Maier M,S., Kommer S and Welzel M (eds) Gender medienkompetent. Medienbildung in Einer Heterogenen Gesellschaft. Wiesbaden: VS-Verlag für Sozialwissenschaften, 111-124.

Parker I (1992) Discourse Dynamics: Critical Analysis for Social and Individual Psychology. London: Routledge.

Parker I (2013) Discourse Analysis: Dimensions of Critique in Psychology. Qualitative Research in Psychology 10(3): 223-239.

Peck BM, Ketchum PR and Embrick DG (2011) Racism and sexism in the gaming world: Reinforcing or changing stereotypes in computer games? Journal of Media and Communication Studies 3(6): 212-220.

Postigo H (2012) The Digital Rights Movement: The Role of Technology in Subverting Digital Copyright. Cambridge: MIT Press.

Postmes T (2007) The psychological dimensions of collective action, online. In: Joinson A, McKena K, Postmes T and Reips U (eds) The Oxford Handbook of Internet Psychology. Oxford: Oxford University Press, 165-184.

Pusch LF (1990) Alle Menschen Werden Schwestern: Feministische Sprachkritik. Frankfurt am Main: Suhrkamp.

Reicher S, Spears R and Haslam SA (2010) The social identity approach in social psychology. In: Wetherell M and Mohanty CT (eds) The SAGE Handbook of Identities. London: SAGE Publications, 45-62.

Rudman LA and Goodwin SA (2004) Gender Differences in Automatic In-Group Bias: Why Do Women Like Women More Than Men Like Men? Journal of Personality \& Social Psychology 87(4): 494-509.

Rust PC (1993) Neutralizing the political threat of the marginal woman: Lesbians' beliefs about bisexual women. Journal of Sex Research 30(3): 214-228.Sauter M (2013) "LOIC Will Tear Us Apart" The Impact of Tool Design and Media Portrayals in the Success of Activist DDOS Attacks. American Behavioral Scientist 57(7): 983-1007.

Söderberg J (2013) Determining social change: The role of technological determinism in the collective action framing of hackers. New Media \& Society 15(8): 1277-1293. 
Stokoe E (2010) 'I'm not gonna hit a lady': Conversation analysis, membership categorization and men's denials of violence towards women. Discourse \& Society 21(1): 59-82.

Tajfel H (1982) Social Identity and Intergroup Relations. Cambridge: Cambridge University Press.

Taylor PA (2003) Maestros or Misogynists? Gender and the Social Construction of Hacking. In: Jewkes Y (ed) Dot. Cons: Crime, Deviance and Identity on the Internet. Cullomptom: Willan, 126-146.

Taylor PA (2005) From hackers to hacktivists: speed bumps on the global superhighway? New Media \& Society 7(5): 625-646.

Thomas D (2003) Hacker Culture. Minnesota: University of Minnesota Press.

Todman J and Day K (2006) Computer anxiety: the role of psychological gender. Computers in Human Behavior 22(5): 856-869.

Turkle S (1984) The Second Self: Computers and the Human Spirit. London: Granada.

Turner JC (1982) Towards a cognitive redefinition of the social group. In: Tajfel H (ed) Social identity and Intergroup Relations. Cambridge: Cambridge University Press, 1540 .

Turner JC, Hogg MA, Oakes PJ, Reicher SD and Wetherell MS (1987) Rediscovering the Social Group: A Self-Categorization Theory. Oxford: Blackwell.

Van Laer J (2007) Internet use and Protest Participation: How do ICTs Affect Mobilization? Antwerpen: Universiteit Antwerpen.

Van Laer J (2010) Activists Online and Offline: the Internet as an Information Channel for Protest Demonstrations. Mobilization 15(3): 347-366.

Van Zomeren M and Iyer A (2009) Introduction to the social and psychological dynamics of collective action. Journal of Social Issues 65(4): 645-660.

Wang H (2009) Language and ideology: gender stereotypes of female and male artists in Taiwanese tabloids. Discourse \& Society 20(6): 747-774. 
Willig C (2008) Introducing Qualitative Research in Psychology: Adventures in Theory and Method. Maidenhead: Open University Press.

Wright SC, Taylor DM and Moghaddam FM (1990) Responding to membership in a disadvantaged group: From acceptance to collective protest. Journal of Personality and Social Psychology 58(6): 994-1003. 


\section{Funding}

This research received no specific grant from any funding agency in the public, commercial, or not-for-profit sector. 